\title{
GPPS-CH-2020-0066
}

\section{DESIGN OF AN AEROFOIL FOR SMALL WIND TURBINES WITH A FOCUS ON TURBULENT INFLOW CONDITIONS}

\author{
Matthias Schmid \\ ForWind - Center for Wind Energy Research \\ Institute of Turbomachinery and Fluid Dynamics \\ Leibniz Universität Hannover \\ schmid@tfd.uni-hannover.de \\ An der Universität 1, 30823 Garbsen, Germany
}

\author{
Joerg R. Seume \\ ForWind - Center for Wind Energy Research \\ Institute of Turbomachinery and Fluid Dynamics \\ Leibniz Universität Hannover \\ seume@tfd.uni-hannover.de \\ An der Universität 1, 30823 Garbsen, Germany
}

\begin{abstract}
Small wind turbines (SWT) must operate deep in the atmospheric boundary layer due to their low hub heights. Turbulence intensities up to $30 \%$ and more are not uncommon in this flow field. This turbulent inflow causes highly variable inflow angles at the rotor blades, even for SWTs which operate at a constant tip speed ratio. An aerofoil design with a high lift-to-drag ratio over a wide range of angles of attack (AoA) is, therefore, beneficial to SWT performance.

A simple analytical method to estimate the size of the AoA variations is presented together with an example design of a new aerofoil shape. The performance of an SWT with this new aerofoil design is evaluated in wind fields with different turbulence intensities using a blade element momentum (BEM) model. The results are compared to an existing SWT aerofoil design. It is shown that this new design methodology yields a performance increase in highly turbulent wind with only minor losses under steady wind conditions.
\end{abstract}

\section{INTRODUCTION}

Wind energy plays a pivotal role in transforming the electric energy system towards renewable energies. The largest proportion of renewable electricity generation in Germany in the year 2017 (about 18\%) was provided by wind turbines. However, sites with good wind conditions are getting rarer, and the resistance against new large wind parks is rising.

SWTs can contribute to the energy transformation. They produce energy even at sites with low average wind speed and can support the energy grid through a distributed energy generation. However, the design process of modern SWTs is not as sophisticated as it is for large wind turbines. The tools used to design SWTs are based on the knowledge gained from large wind turbines, but SWTs experience different inflow conditions and the requirements diverge from those of large wind turbines. In general, the operational wind speeds of small wind turbines are considerably lower, and the incoming wind is much more turbulent.

The design of aerofoils for SWTs had been neglected for a long time and instead aerofoils intended for large wind turbines or aeroplanes were used. In recent years, some publications have developed aerofoils for SWTs and demonstrated the performance increase of SWTs offered by using tailored aerofoil shapes, e.g. (Giguere and Selig, 1998; Henriques et al., 2009; Kim et al., 2010; Leloudas et al., 2020; Sanaye and Hassanzadeh, 2014; Singh et al., 2012; Wata et al., 2011).

In this paper, a method to design an SWT aerofoil shape is introduced with a focus on the turbulence of the inflow. This turbulence imposes large variations in the inflow angle even for wind turbines, which operate at a constant tip speed ratio (variable speed). An example aerofoil is presented, and the performance of an SWT with this example aerofoil is compared to an SWT with an existing aerofoil shape obtained from literature. This comparison is performed at different turbulence intensities using two blade element momentum models. 


\section{METHODOLOGY}

The design of a tailored aerofoil shape for SWTs requires some boundary conditions to be determined first, these consist of the blade geometry and wind conditions.

A new blade geometry can be derived by using Glauert's theory as described in (Hansen, 2015). The equations for the inflow angle $\varphi(r)$ and chord distribution $\mathrm{c}(\mathrm{r})$ are:

$$
\begin{gathered}
\phi(r)=\arctan \left(\frac{(1-a(r)) R}{\left(1+a^{\prime}(r)\right) \lambda r}\right) \\
c(r)=\frac{8 \pi F(r) a(r) r \sin (\phi(r))^{2}}{(1-a(r)) B c_{n}(r)}
\end{gathered}
$$

Here $a(r)$ and $a^{\prime}(r)$ are the axial and tangential induction factors, respectively. $\lambda$ is the tip speed ratio, $r$ the radial position, $\mathrm{R}$ the rotor blade radius, $\mathrm{F}(\mathrm{r})$ the tip loss factor, $\mathrm{B}$ the number of blades and $\mathrm{c}_{\mathrm{n}}(\mathrm{r})$ the coefficient of lift and drag normal to the rotor plane and can be calculated with:

$$
c_{n}(r)=c_{l} \cos (\phi(r))+c_{d} \sin (\phi(r))
$$

where $c_{1}$ and $c_{d}$ are the lift and drag coefficients of the aerofoil, respectively.

To use this method, the following design parameters, must be specified: number of blades B, rotor radius R, tip speed ratio $\lambda$, lift coefficient $c_{1}$ and drag coefficient $c_{d}$. The new aerofoil design is meant to be a re-design of a reference wind turbine located in northern Germany, therefore, the number of blades, rotor radius, and tip speed ratio will be kept constant. At this point, a design lift and drag coefficient can only be roughly estimated as they depend on the aerofoil shape which is yet to be designed. The optimum chord distribution, according to Glauert, results for the given tip speed ratio in relatively large chord lengths near the root. A high lift coefficient of 1.2 is, therefore, favoured to reduce these large chord lengths. To estimate the drag coefficient, a lift-to-drag ratio of 100 is used. This value should be achievable for a good aerofoil design, and results in a design drag coefficient of 0.012 .

The determination of the prevailing wind conditions is based on measurement data from the reference site and can be seen in form of a histogram of the 10-min mean wind speeds in Figure 1. The fit of the Weibull distribution (shape parameter $=1.78$, scale parameter $=5.71$ ) is also plotted and shows a good agreement with the measured data. The overall mean wind speed at this site is about $5 \mathrm{~m} / \mathrm{s}$, which is chosen as design wind speed.

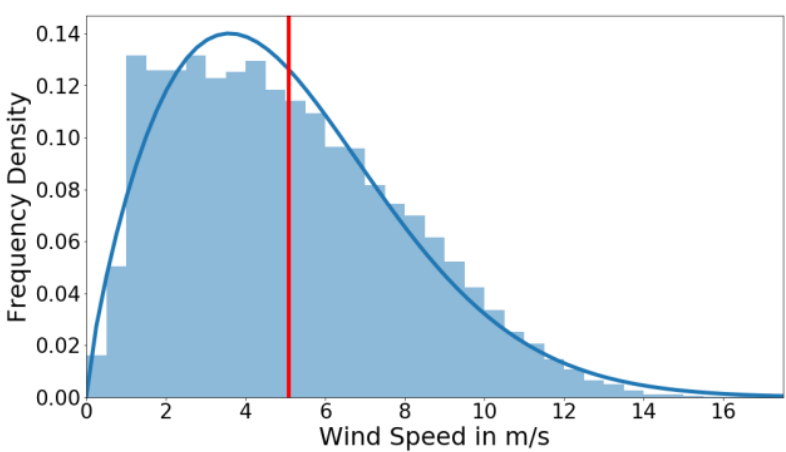

Figure 1 Histogram of the 10-min mean wind speeds measured at the reference site, together with a Weibull fit and the overall mean wind speed

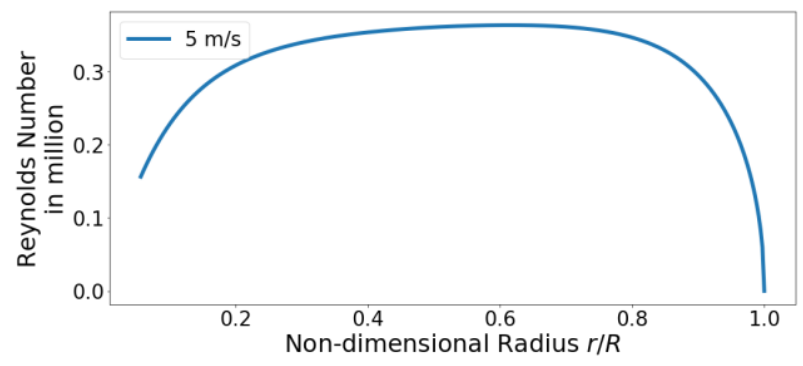

Figure 2 Distribution of the Reynolds number along the rotor blade span at a wind speed of $5 \mathrm{~m} / \mathrm{s}$ using the chord distribution according to Eqn. (2)

Using the original tip speed ratio, the derived chord distribution and induction factors calculated with Glauert's theory the Reynolds number for the design wind speed can be calculated. The distribution of this Reynolds number along the rotor blade span is plotted in Figure 2. It can be observed that the Reynolds number for the design wind speed is slightly above 300,000 along most of the rotor blade span, except for the root and tip regions due to secondary flows at the root and the tip modelled by a Prandtl tip loss factor. Therefore, a design Reynolds number of 300,000 is chosen.

A closer look on the inflow conditions shows that SWTs must operate in a highly turbulent wind. SWTs are deeper in the atmospheric boundary layer due to their low hub heights, which results in lower mean wind speeds and higher turbulence intensities. Also, the siting of SWTs in the vicinity of obstacles like buildings or vegetation, which is sometimes required, increases these difficulties. The turbulence intensities of different mean wind speeds are plotted in Figure 3. In this plot, the guideline according to the standard for small wind turbines (IEC 61400-2 International Electrotechnical Commission, 2013) is plotted together with multiple measurements. For a wind speed of $5 \mathrm{~m} / \mathrm{s}$, a value 
for the turbulence intensity of $30 \%$ is recommended by the standard. This recommendation increases strongly for lower wind speeds and decreases only slightly for higher wind speeds. The measurements from the reference site show considerably lower turbulence intensities. This can be explained by the sampling rate of only 1 sample per 10 seconds because, at this rate, fast turbulent fluctuations cannot be captured, and thus the resulting turbulence intensity is too low. Other more precise measurements, for example from the Alaiz Experiment by (Santos et al., 2019), show higher turbulence intensities, which are, depending on the actual siting, within the range of the recommendations of the standard. For the further design process, a turbulence intensity of $25 \%$ is used, which is slightly below the standard and fits to the measurements.

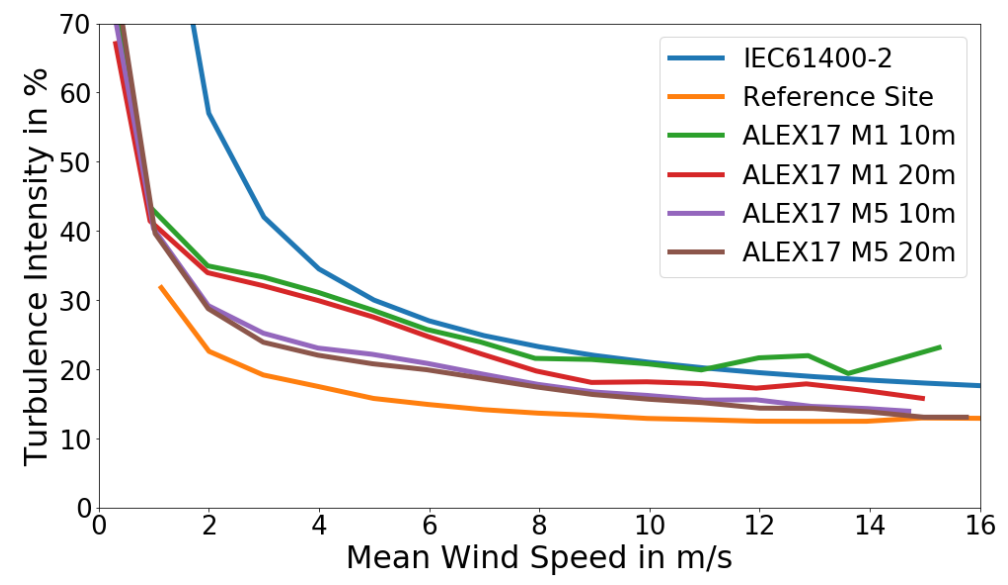

\section{Figure 3 Turbulence intensity versus mean wind speed derived from different measurements and according to the standard for small wind turbines}

A Weibull distribution approximates the distribution of the 10-min mean wind speeds very well as shown in Figure 1. Conversely, the distribution of the instantaneous wind speeds during a 10 min interval is unknown and, therefore, a normal distribution with $5 \mathrm{~m} / \mathrm{s}$ as mean and the standard deviation derived from the turbulence intensity is used further in the process. Analysing this distribution, results in a range of wind speeds from $2.9 \mathrm{~m} / \mathrm{s}$ to $7.1 \mathrm{~m} / \mathrm{s}$ if $90 \%$ of the wind speeds should be considered.

This large range of instantaneous wind speeds has an influence on the angle of attack, which an aerofoil on an SWT experiences. The variation of the inflow angle can be estimated at constant rotational speed because the rotor of a variable-speed wind turbine is not able to follow the fast changes of the wind speed during turbulent fluctuations, due to forces of inertia. The resulting variations of the inflow angle $\varphi$ are shown in Figure 4. There, the inflow angle calculated with Eqn. (1) is shown together with the range of inflow angles, which occur for wind speeds between $2.9 \mathrm{~m} / \mathrm{s}$ and 7.1 $\mathrm{m} / \mathrm{s}$ at constant rotational speed of the rotor. For the outer part of the rotor blade (from $75 \%$ to the tip) a maximum deviation of the inflow angle and, therefore, the angle of attack from $-3.9^{\circ}$ to $+3.7^{\circ}$ can be observed.

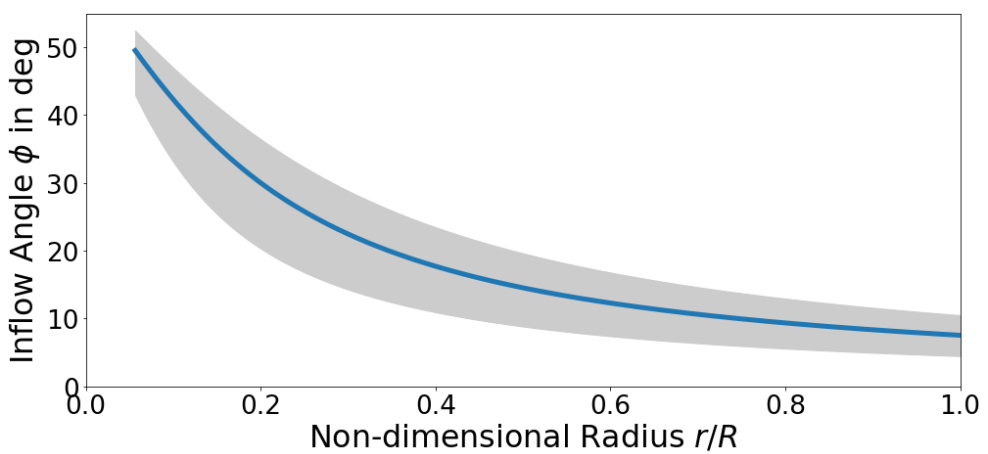

Figure 4 Range of inflow angles occurring along the blade span for a mean wind speed of $5 \mathrm{~m} / \mathrm{s}$ with a turbulence intensity of $25 \%$ and constant rotational speed

This emphasises the large variations of the angle of attack which an aerofoil experiences even under normal wind conditions. The effect of gusts, wind direction changes or other extreme conditions are not yet considered. The design of an aerofoil shape even for a variable-speed wind turbine operating at a constant tip speed ratio, therefore, must accommodate a wide range of angles of attack. 


\section{RESULTS AND DISCUSSION}

The results of this paper are twofold. First, an example aerofoil design is presented, which takes the turbulent nature of the incoming wind into account. Second, the performance of an SWT with this new aerofoil is compared to an SWT with an existing aerofoil obtained from the literature. For the latter, simulations with two blade element momentum models have been conducted.

The main requirement of an aerofoil shape is a high lift-to-drag ratio, as this ratio is beneficial to the rotor performance. Despite the fact that the reference wind turbine is a variable-speed turbine and operates at a nearly constant tip speed ratio (and hence the rotor aerofoils at a constant angle of attack), the range of angle of attacks where a high liftto-drag ratio is required should be relatively wide due to the highly fluctuating wind speeds as shown in the previous section. The highest lift-to-drag ratio should occur at a lift coefficient of about 1.2, which was chosen for the derivation of the blade geometry. To increase the lift-to-drag ratio, the drag must be reduced and, therefore, the presence of laminar separation bubbles should be avoided for Reynolds numbers at and above the design Reynolds number. Additionally, the aerofoil should not be sensitive to leading edge roughness as this can occur during the long operational time of a small wind turbine.

A design tool developed by (Eppler, 2012) has been used (version 18.1.08) to tailor a new aerofoil shape. This tool uses an inverse design methodology based on conformal mapping. The desired velocity/pressure distributions along the upper and lower surface must be prescribed and the design tool calculates the corresponding aerofoil shape. Predefined velocity distributions are available for the main pressure recovery regions, and an iterative process can be used to assure a closed profile shape.

The velocity distributions are chosen in order to obtain a low drag value of the aerofoil shape. One of the main sources of drag in this low Reynolds number regime is the presence of laminar separation bubbles. To avoid these separation bubbles only a slight pressure increase (and, therefore, a slight velocity decrease) is possible over the whole chord length. A large leading edge radius is also included to avoid large suction peaks at the leading edge as they also induce a large pressure increase and, therefore, the danger of laminar separation bubbles of complete separation.

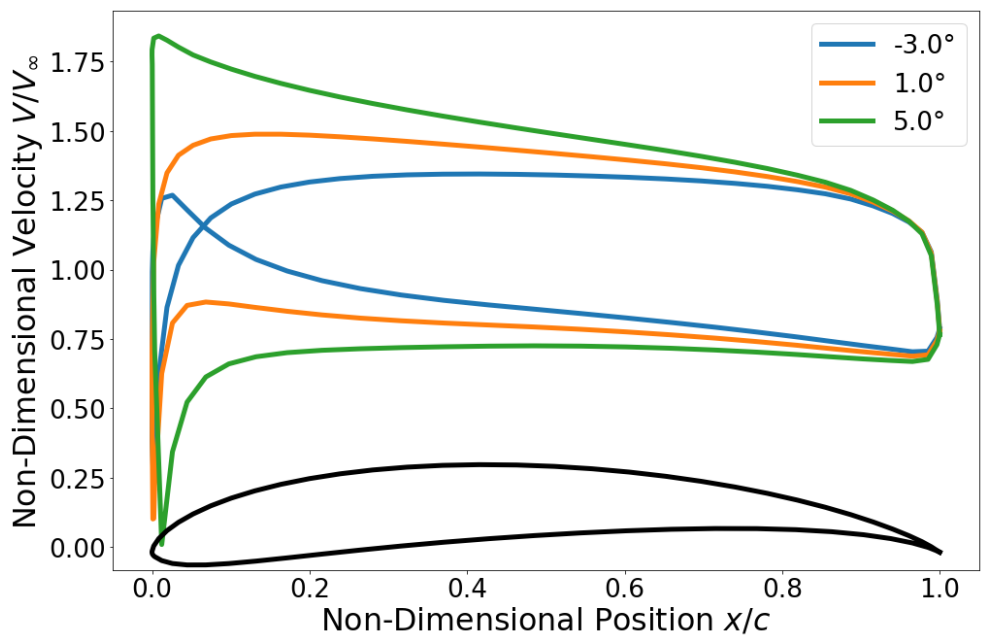

Figure 5 Geometry of new aerofoil and velocity distributions along the chord for three different angles
of attack

Figure 5 shows the profile shape together with three velocity distributions along the upper and lower surfaces for the angles of attack of $-3^{\circ}, 1^{\circ}$, and $5^{\circ}$. This aerofoil reaches its highest lift-to-drag ratio at an AoA of $1^{\circ}$ (optimum AoA), the AoA of $-3^{\circ}$ and $5^{\circ}$ correspond, therefore, to the limits of the AoA range considered during the design process. All velocity distributions are calculated with the Eppler tool. Within this tool, it is only possible to account for a turbulent inflow by reducing the $\mathrm{N}$-factor of the boundary layer transition, therefore, the default value of 11 has been reduced to a value of 5 . This models the influence of the turbulence only on the boundary layer transition in a very basic way, other effects of the highly turbulent inflow cannot be modelled within this tool and further investigations with higher-order tools are planned in future. It can be seen, for the plotted range of angles of attack that on the upper and on the lower surface only a small pressure increase is present along almost the whole chord length and suction peaks are nearly avoided. This behaviour is favourable in this low Reynolds number regime, as the laminar boundary layer is only capable to withstand a slight pressure increase without separation.

The new designed aerofoil has at an AoA of $1^{\circ}$ a maximum lift-to-drag ratio of 105 and a lift coefficient of 1.201 . Compared to the aerofoils SG6041, SG6042 and SG6043 by (Giguere and Selig, 1998) the new aerofoil has a higher camber and, therefore, higher lift coefficients at the same angle of attack. The maximum lift-to-drag ratio also occurs at a lower angle of attack, as can be seen in Figure 6. The value of the maximum lift-to-drag ratio is slightly lower than for 
the SG6043, but the decrease of the lift-to-drag ratio at angles of attack next to the optimum is lower than for the SG aerofoils. Especially for positive offsets a significant improvement could be realised, for negative offsets the improvement is smaller as can be seen in Table 1. In Table 1 the reduction of the lift-to-drag ratio in percent from the maximum lift-to-drag ratio is given for different delta angles of attack, where the delta denotes the distance from the optimum angle of attack.

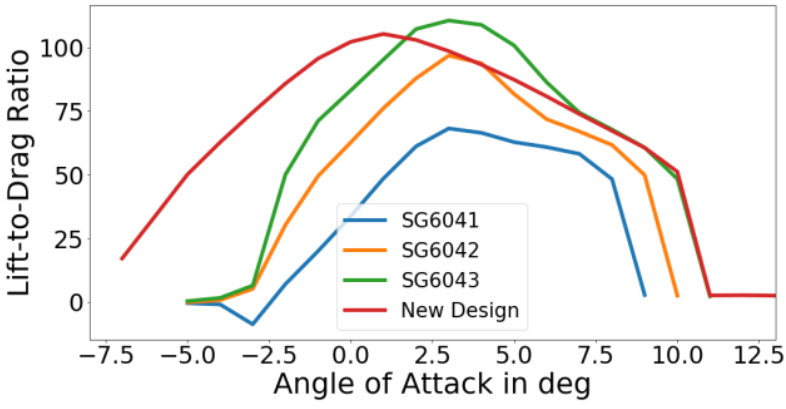

Figure 6 Lift-to-drag ratio against the angle of attack for the new aerofoil and three aerofoils designed by (Giguere and Selig, 1998)

\begin{tabular}{l|cccc} 
Delta AoA & $-4^{\circ}$ & $-2^{\circ}$ & $2^{\circ}$ & $4^{\circ}$ \\
\hline SG6043 & $-35.6 \%$ & $-13.9 \%$ & $-8.8 \%$ & $-32.7 \%$ \\
New Design & $-29.2 \%$ & $-9.2 \%$ & $-6.4 \%$ & $-17.0 \%$
\end{tabular}

Table 1 Reduction of lift-to-drag ratio at different delta angles of attack relative to optimum (highest lift-to-drag ratio)

To assess the performance of the new aerofoil design on an SWT a simple model of a wind turbine has been set up with the OpenFAST software, which is described by (Jonkman and Sprague, 2018). With this model the performance of the new aerofoil design can be compared to other aerofoils in steady and turbulent wind. The model setup uses four OpenFAST modules: AeroDyn, InflowWind, ServoDyn and ElastoDyn.

In the AeroDyn (v15) module the aerofoil polars, the chord and twist distributions are prescribed. This module uses the dynamic wake/induction model (DBEMT) to account for the turbulent fluctuations of the wind speed. For the blade aerofoil aerodynamics the steady model has been chosen, as no dynamic aerofoil data is available.

The aerofoil polars of the SG aerofoils and the new aerofoil design were analysed with the Eppler code to get the lift and drag coefficient in the linear region and up to the maximum lift coefficient. For higher angles of attack (up to $180^{\circ}$ ) the tool airfoilPreppy from (Ning, 2014) was used. This tool extrapolates the lift and drag coefficients based on the Viterna method, which approximates the aerofoil polars at high angles of attack based on flat plate aerodynamics. Dynamic aerofoil properties are not included in these polar data as the tools used are not capable to produce these information.

The rotor blade chord distribution differs little for the two different aerofoils because of the different lift and drag coefficients at maximum lift-to-drag ratio. Using the obtained lift and drag coefficients from the Eppler tool the chord distribution is calculated according to Eqn. (2). The optimum twist angle of the rotor blades also differs for the two different aerofoils, because of the different optimum angle of attack and is derived from Eqn. (1).

The wind conditions are defined in the InflowWind module. For the steady, case a horizontal wind speed of $5 \mathrm{~m} / \mathrm{s}$ has been used at a reference height equal to the hub height. The profile of the incoming wind obeys the power law with an exponent of 0.2. For the turbulent cases, the incoming wind field has been generated with TurbSim from (Kelley and Jonkman, 2016), also with a mean wind speed of $5 \mathrm{~m} / \mathrm{s}$ at hub height. The IECKAI model (International Electrotechnical Commission, 2005) has been used as turbulence model with different characteristic turbulence values between $0 \%$ and $30 \%$. For both SWT models, the same wind input files has been used to ensure equal conditions.

The characteristics of the generator are prescribed in the ServoDyn module by a speed-torque curve. This curve has been generated based on the desired constant tip speed ratio and an estimation of the rotor torque by using the following equations:

$$
\begin{gathered}
c_{t}(r)=c_{l} \sin (\phi(r))-c_{d} \cos (\phi(r)) \\
v_{n}(r)=v_{0}(1-a(r)) \\
v_{t}(r)=\left(1+a^{\prime}(r)\right) v_{0} \lambda \frac{r}{R} \\
T=B \int_{r_{i}}^{R} \frac{\rho}{2}\left(v_{n}(r)^{2}+v_{t}(r)^{2}\right) c(r) c_{t}(r) r d r
\end{gathered}
$$

Where $v_{0}$ is the undisturbed wind speed, $\rho$ the density, and $r_{i}$ is the blade root radius. This torque value has been calculated for multiple wind velocities and, therefore, with constant tip speed ratio for different rotational speeds. The 
results of these calculations are then stored in a table and used as generator torque to maintain a constant tip speed ratio during the simulations.

In the ElastoDyn module only the generator degree of freedom is activated to allow the rotor adjust its speed according to the prevailing wind conditions. The rotor blades and the wind turbine tower are modelled as stiff structures with no bending. Both wind turbine models have the same mass distributions.

The steady case has been simulated for $30 \mathrm{~s}$ with a time step size of $0.001 \mathrm{~s}$ and an initial rotational speed as expected by the fixed tip speed ratio. The turbulent cases have been started with the same initial rotational speed and time step size. The duration has been set to $610 \mathrm{~s}$ whereas the first ten seconds have been neglected in the analysis. The rotational speed during all simulations has not been prescribed and adjusted itself according to the prevailing aerodynamic and generator torque. In total two small wind turbine models have been set up, one with the new aerofoil design and the second with the SG6043 aerofoil.

\begin{tabular}{l|ccccc} 
TI in \% & 10 & 15 & 20 & 25 & 30 \\
\hline Min. wind speed in m/s & 4.3 & 3.9 & 3.5 & 3.1 & 2.8 \\
Max. wind speed in m/s & 5.9 & 6.3 & 6.7 & 7.1 & 7.6
\end{tabular}

Table 2 Min. and max. wind speeds for different turbulence intensities derived from the $5^{\text {th }}$ and $95^{\text {th }}$ percentile

As a means of verification, the axial wind speed in the BEM simulations has been analysed. The mean wind speed is almost constant over all simulations with a slight decrease at higher turbulence intensities (minimum $4.946 \mathrm{~m} / \mathrm{s}$ ). In Table 2 are the $5^{\text {th }}$ and $95^{\text {th }}$ percentiles of the wind speed for the turbulent test cases listed. These values represent the upper and lower limit of $90 \%$ of the wind speeds. The estimation based on a normal distribution in the previous section resulted in values of $2.9 \mathrm{~m} / \mathrm{s}$ and $7.1 \mathrm{~m} / \mathrm{s}$ for a turbulence intensity of $25 \%$. The upper limit met the estimation and the lower limit is only slightly higher $(0.2 \mathrm{~m} / \mathrm{s})$ than estimated. This shows, that the simulated wind field meets the expected properties.

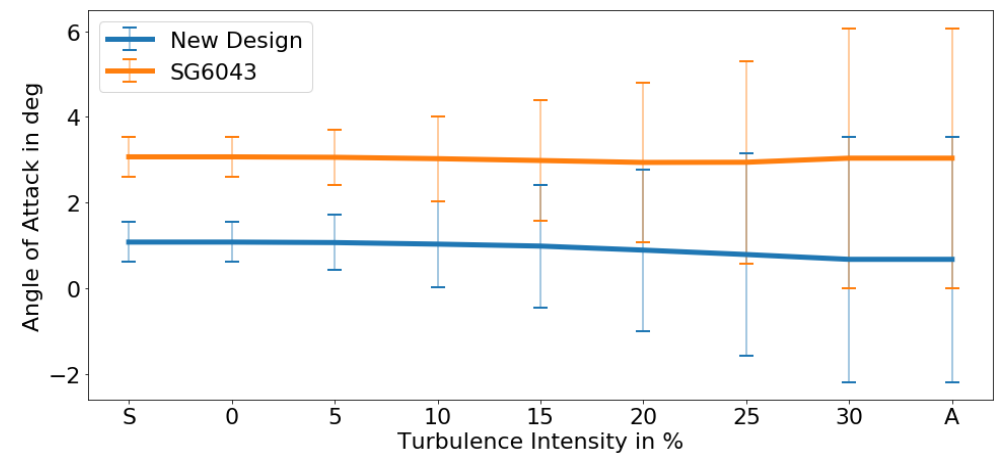

Figure 7 Mean angle of attack near $75 \%$ rotor radius for different turbulence intensities and the standard deviation indicated by the error bars

The angles of attack extracted from the two BEM simulations of a rotor blade section near $75 \%$ radius are shown in Figure 7. The value $\mathrm{S}$ for the turbulence intensity represents the steady test case and is equal with the zero turbulence case. The value A represents a turbulence as defined in the IEC 61400-2 and is equal to the test case with $30 \%$ turbulence intensity. The angles of attack for the different simulations are almost constant at the optimum value of $3^{\circ}$ and $1^{\circ}$ for the SG6043 and the new designed aerofoil, respectively. As expected, the range of angles of attack grows with increasing turbulence intensity indicated by the error bars, which represent the standard deviation of the angle of attack. The angle of attack has been estimated to vary in a range from $-3.9^{\circ}$ to $+3.7^{\circ}$ around the optimum angle. In Table 3 , the deviations from the nominal angle of attack, which occur during the BEM simulations are listed. These values are also obtained using the $5^{\text {th }}$ and $95^{\text {th }}$ percentile of the angles of attack. The estimated range of the angle of attack fits very good to the actual values listed in Table 3 for a turbulence intensity of $25 \%$.

\begin{tabular}{l|ccccc}
$\mathrm{TI}$ in $\%$ & 10 & 15 & 20 & 25 & 30 \\
\hline Min. delta AoA in $^{\circ}$ & -1.6 & -2.4 & -3.3 & -4.2 & -5.2 \\
Max. delta AoA in $^{\circ}$ & 1.7 & 2.4 & 2.9 & 3.7 & 4.2
\end{tabular}

Table $3 \mathrm{Min}$. and max. deviations from the nominal angle of attack at $75 \%$ rotor radius for different turbulence intensities derived from the $5^{\text {th }}$ and $95^{\text {th }}$ percentile 
The mean value of the angle of attack is also shown in Figure 8. In this plot the angle of attack is plotted against the rotor blade radius for four different turbulence intensities. The largest deviations of the mean angle of attack occur near the blade root where the tangential velocity due to the rotational speed of the rotor is the lowest. It can also be seen, that for the new aerofoil design the deviations of the angle of attack from its optimum value are larger than for the SG6043 aerofoil.

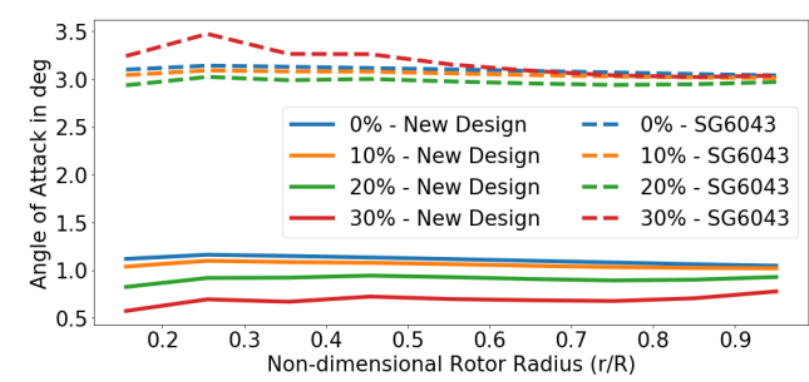

Figure 8 Local mean angle of attack against rotor radius for four different turbulence intensities

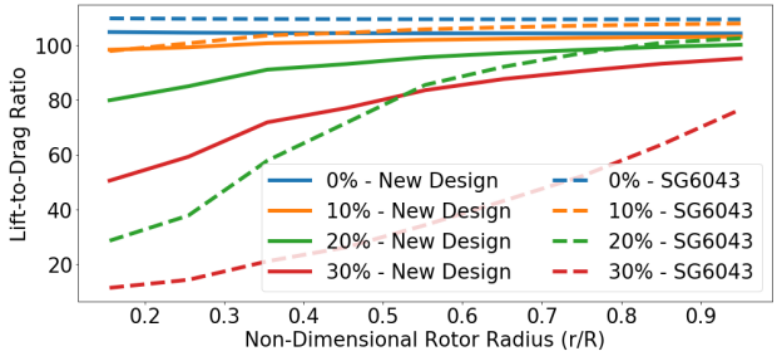

Figure 9 Local mean lift-to-drag ratio against rotor radius for four different turbulence intensities

The effect of these angle of attack deviations on the local lift-to-drag ratio can be seen in Figure 9, where the lift-todrag ratio is plotted against the rotor blade radius for the same four turbulence intensities. The general trend of a reduced lift-to-drag ratio with higher turbulence intensity can be recognised. For the low turbulence intensities the local lift-todrag ratio of the SG6043 aerofoil is higher than for the new aerofoil design. For higher turbulence intensities the new aerofoil design can achieve higher local lift-to-drag ratios than the SG6043 aerofoil despite the larger angle of attack deviations seen in Figure 8.

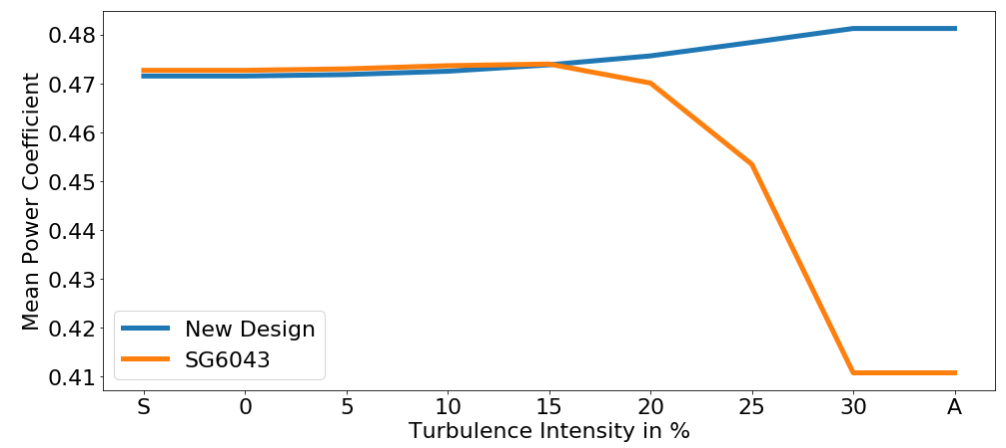

\section{Figure 10 Mean power coefficient for different turbulence intensities for the new aerofoil and the SG6043}

In Figure 10, the mean power coefficients for both wind turbine models are plotted against the different turbulence intensities of the wind. These power coefficients are calculated at every time step and then averaged. The power coefficient increases slightly with increasing inflow turbulence for both wind turbine models up to a turbulence intensity of $15 \%$. This slight increase of the power coefficient can be explained with the higher total wind speeds at higher turbulence intensities. The axial component of the wind speed is kept constant at $5 \mathrm{~m} / \mathrm{s}$ but for higher turbulence intensities the other wind components increase which can also contribute to the power extraction. Up to $15 \% \mathrm{TI}$, the wind turbine model with the SG6043 aerofoil shows a small benefit compared the model with the new aerofoil design. This small performance benefit can be explained with the higher optimum lift-to-drag ratio of the SG6043 aerofoil. For higher turbulence intensities from $15 \%$ to $30 \%$, the mean power coefficient of the new aerofoil design continues to increase, whereas, the mean power coefficient of the SWT with the SG6043 starts to drop. At these higher turbulence intensities, the lift-to-drag ratios next to the optimum value becomes more important as the angle of attack varies strongly. Here the new aerofoil design improves the SWT performance. At $25 \%$ turbulence intensity the mean power coefficient is increased by $5.5 \%$ relative to the SG6043 aerofoil and at 30\% turbulence intensity it is increased by $17.1 \%$.

\section{CONCLUSIONS}

The aim of this investigation was to develop and assess a simple design process, which can account for the turbulent nature of the incoming wind for small wind turbines. It could be shown that turbulence intensities up to $30 \%$ are not uncommon for small wind turbines and this high turbulence causes large variations of the angle of attack during normal operation. Even small wind turbines operating at a constant tip speed ratio must consider the turbulence of the incoming 
wind. A simple estimation for the range of angles of attack was verified with a blade element momentum model of a small wind turbine. The rotor blade sections experience similar wind conditions and angles of attack during the simulation as estimated before with simple analytic equations.

A new aerofoil shape has been presented, which provides a slightly lower optimum lift-to-drag ratio compared to other small wind turbine aerofoils obtained from literature but offers higher lift-to-drag ratios next to the optimum angle of attack. The mean power coefficient for both small wind turbine models (with the new aerofoil design and the SG6043 aerofoil) increases with increasing turbulence intensity up to a value of $15 \%$. For higher turbulence intensities, the mean power coefficient drops for the SG6043 aerofoil, however, continues to increase for the new aerofoil design. This can be explained by the higher lift-to-drag ratios next to the optimum AoA as these become more important under turbulent wind conditions. Therefore, under highly turbulent conditions a performance increase of up to $17.1 \%$ can be achieved using the new design procedure.

Some uncertainties in the aerofoil performance calculation must be investigated further. These affect the calculated lift and drag coefficients of the aerofoils. A scale resolving simulation and experiments are currently planned to validate the calculated aerofoil polars and possibly to capture the influence of the inflow turbulence also on boundary layer transition.

\section{NOMENCLATURE}

\begin{tabular}{|c|c|c|c|}
\hline $\mathrm{a}$ & Axial induction factor & $\mathrm{v}_{0}$ & Wind speed \\
\hline$a^{\prime}$ & Tangential induction factor & $\mathrm{v}_{\mathrm{n}}$ & Component of the relative wind speed normal to \\
\hline B & Number of blades & & the rotor plane, Eqn. (5) \\
\hline $\mathrm{C}$ & Chord length, Eqn. (2) & $\mathrm{V}_{\mathrm{t}}$ & Component of the relative wind speed tangential \\
\hline$c_{d}$ & Drag coefficient & & in the rotor plane, Eqn. (6) \\
\hline$c_{l}$ & Lift coefficient & $\phi$ & Inflow angle, Eqn. (1) \\
\hline$c_{n}$ & Coefficient normal to rotor plane, Eqn. (3) & $\lambda$ & Tip speed ratio \\
\hline$c_{t}$ & Coefficient tangential in rotor plane, Eqn. (4) & $\rho$ & Density of air \\
\hline $\mathrm{F}$ & Tip loss correction factor & AoA & Angle of attack \\
\hline r & Radial position & BEM & Blade element momentum \\
\hline$r_{i}$ & Root radius & $\operatorname{Re}$ & Reynolds \\
\hline $\mathrm{R}$ & Rotor (tip) radius & SWT & Small wind turbine \\
\hline $\mathrm{T}$ & Rotor torque, Eqn. (7) & $\mathrm{TI}$ & Turbulence intensity \\
\hline
\end{tabular}

\section{References}

Eppler, R. (2012). Airfoil design and data. Springer Science \& Business Media.

Giguere, P. and Selig, M.S. (1998). New airfoils for small horizontal axis wind turbines. Journal of solar energy engineering, 120.2, pp.108-114.

Hansen, M. O. (2015). Aerodynamics of wind turbines. Routledge.

Henriques, J., Marques da Silva, F. Estanqueiro, A.I., and Gato, L. (2009). Design of a new urban wind turbine airfoil using a pressure-load inverse method. Renewable Energy, 34.12, pp. 2728-2734.

International Electrotechnical Commission et al. (2005). IEC 61400-1: Wind turbines-part 1: Design requirements. International Electrotechnical Commission et al. (2013). IEC 61400-2: Wind turbines - part 2: small wind turbines.

Jonkman, J. and Sprague, M. (2018). NWTC Information Portal (OpenFAST). Available at: https://nwtc.nrel.gov/OpenFAST. [Accessed: 2019-12-16]

Kelley, N. and Jonkman, B. (2016). NWTC Information Portal (TurbSim). Available at: https://nwtc.nrel.gov/TurbSim. [Accessed: 2019-12-16]

Kim, T., Lee, S., Kim, H., and Lee, S. (2010). Design of low noise airfoil with high aerodynamic performance for use on small wind turbines. Science China Technological Sciences, 53.1, pp. 75-79.

Leloudas, S. N., Eskantar, A. I., Lygidakis, G. N., and Nikolos, I. K. (2020). Low Reynolds airfoil family for small horizontal axis wind turbines based on RG15 airfoil. SN Applied Sciences, 2.3.

Ning, S. A. (2014). NWTC Information Portal (AirfoilPrep.py). Available at: https://nwtc.nrel.gov/AirfoilPrepPy. [Accessed: 2019-12-16]

Sanaye, S. and Hassanzadeh, A. (2014). Multi-objective optimization of airfoil shape for efficiency improvement and noise reduction in small wind turbines. Journal of Renewable and Sustainable Energy, 6.5, p. 053105

Santos, P., Mann, J., Vasiljevic, N., Courtney, M., Sanz Rodrigo, J., Cantero, E., et al. (2019). The Alaiz Experiment (ALEX17): wind field and turbulent fluxes in a large-scale and complex topography with synoptic forcing. Technical University of Denmark. Collection

Singh, R. K., Ahmed, M. R., Zullah, M. A., and Lee, Y.-H. (2012). Design of a low Reynolds number airfoil for small horizontal axis wind turbines. Renewable Energy, 42, pp. 66-76.

Wata, J., Faizal, M., Talu, B., Vanawalu, L., Sotia, P., and Ahmed, M. R. (2011). Studies on a low Reynolds number airfoil for small wind turbine applications. Science China Technological Sciences, 54.7, pp. 1684-1688. 